\title{
Statin-Associated Autoimmune Myopathy: Diagnostic Challenges
}

\author{
Nirosen Vijiaratnam ${ }_{\text {MBBS, BMedSci }}{ }^{1}$, Matthew Jiang ${ }^{1}$ MBBS, BmedSci, Tissa Wijeratne ${ }^{1,2}{ }_{\text {FRACP }}$ \\ ${ }^{1}$ Department of Neurology, Western Health, Gordon Street, Footscray 2011 \\ ${ }^{2}$ The University of Melbourne
}

\section{Keywords:}

- Statin myopathy is a heterogenous condition

- Diagnosing statin-associated autoimmune myopathy is challenging but critical

- Anti HMGCR autoantibodies are useful in making the diagnosis

- Immunosuppresion is critical in this condition

\section{CLINiCAL RECORD}

We report an interesting case of a 63 year old lady of Middle Eastern background who presented to our hospital with a 3 day history of acute onset fatigue, muscle pain and severe weakness in her lower limbs. She had several episodes of diarrhoea with nausea and vomiting 2 days prior to her presentation, but no fevers or chills and no rashes. There was no history of trauma or falls or prolonged periods of immobilisation.

Her past history included a 10 year history of type 2 diabetes, ischaemic heart disease with previous percutaneous coronary intervention; stage 3 chronic kidney disease, dyslipidaemia, depression, previous gastric banding surgery, appendicectomy and cholecystectomy.

Her medications included irbersartan, diltiazem, rosuvastatin, ezetimibe, metformin, clopidogrel, fluoxetine, esomeprazole and seretide.

She had been on rosuvastatin for over 15 years and has had no side effects to the medication. Her dose had not been altered for a number of years.

On examination, she was drowsy but oriented with normal vital signs. Her cardiorespiratory and gastrointestinal examination was unremarkable. She had normal tone, severe weakness in hip flexion bilaterally, with normal power in other muscle groups. Her reflexes were symmetrical and normal and her sensory exam was unremarkable. Her upper limbs and cranial nerves were unremarkable.

Her investigations showed a metabolic acidosis $\left(\mathrm{pH} 7.18, \mathrm{HCO}_{3} 11 \mathrm{mmol} / \mathrm{L}, \mathrm{pCO}_{2} 30 \mathrm{mmHg}\right)$ with anuric renal failure (creatinine $700 \mu \mathrm{mol} / \mathrm{L}, \mathrm{eGFR} 5 \mathrm{~mL} / \mathrm{min}$, Urea $32.9 \mathrm{mmol} / \mathrm{L}, \mathrm{K} 6.5 \mathrm{mmol} / \mathrm{L}$ ) and deranged LFTs (AST 1299 ALT 582, ALP \&GGT normal, bilirubin $2 \mu \mathrm{mol} / \mathrm{L}$ ). Her CK was 55,464U/L. Her troponin and ECG were normal.

She underwent haemofiltration and was started on IVIg $(0.4 \mathrm{mg} / \mathrm{kg} /$ day for 5 days $)$ and pulse methylprednisolone ( $1 \mathrm{~g}$ daily for 3 days) for presumed statin associated autoimmune myopathy. Her rosuvastatin, ezetemibe and diltiazem were ceased.

The initial EMG sampling of right vastuslataralis revealed reduced voluntary activation with no evidence of membrane irritability, myopathic changes or denervation changes. The study was repeated 3 weeks later and was normal.

A muscle biopsy was taken from her left vastuslateralis after the first day of methylprednisolone. There was no inflammatory cell infiltrate, no vasculitis and only occasional degenerating fibres. Phosphorylase and AMP deaminase was normal and there was no abnormal cytochrome oxidase depletion. Severe lipid overload was noted. This was consistent with a statin induced change.

\footnotetext{
${ }^{1}$ Corresponding Author: nirosenv@gmail.com
} 
Secondary causes were also ruled out. Her thyroid function tests, hepatitis serology (A,B, C), autoimmune screen (dsDNA, ENA, ANCA and anti-GBM), serum protein electrophoresis and light chain ratio were all normal.

Her anti-HMG-CoA reductase antibody was later found to be negative, with an antibody titre of 0.07 (ref $<0.24$ ).

She was discharged from ICU after 3 days and remained on the ward for another month with recovering renal function on haemodialysis. Her CK was $17,000 \mathrm{U} / \mathrm{L}$ with ongoing proximal muscle weakness on day 6 . Her admission was complicated by delirium with several urinary tract infections and required a period of rehabilitation where she stayed for 9 days. Her CK slowly improved back to normal over 20 days. On admission to rehabilitation she was noted to have $4 / 5$ power in bilateral hip flexion, with normal power in all other movements. She continued to require assistance standing. On discharge she was able to walk independently but had ongoing proximal weakness.

She continued monthly IVIG. Her most recent CK was 54U/L and creatinine had improved to $106 \mu \mathrm{mol} / \mathrm{L}$. She was last reviewed about 5 months post discharge. Her power was 5-/5 in both hip flexion. She finds that her weakness is worse approximately 2 weeks after her IVIG doses. The plan is to gradually wean her off the IVIG.

\section{DISCUSSION}

Statins are amongst the most widely prescribed class of drugs in routine clinical practice. They are highly effective but their side effects limit use in about $5-20 \%$ of patients. $(1,2)$ The most common side effect is musculoskeletal predominantly pain but myopathy and rhabdomyolyis is well recognised. Statin myopathy is a self-limiting condition on cessation of the medication. Statins can uncommonly cause a severe, fulminant immune mediated myopathy which does not resolve on cessation of the medication and responds well to immunomodulatory therapy (3)

Whilst making the distinction is critical, it can often be challenging. The first point to be made is, whilst self-limited statin myopathy resolves once the offending medication is removed, it can take between 1 week and 14 months to resolve and therefore can't be used solely as a distinguishing factor. (4) Atorvastatin, Pravastatin and Simvastatin have all been reported as causes for immune mediated myopathy. (5) There is no data to our knowledge of Rosuvastatin being a cause of autoimmune myopathy, which makes this case unique. The longer duration of statin use prior to presentation would point towards an immune mediated myopathy. Median duration for a self-limited myopathy is 6.3 months with a range from 1 week to 4 years in one report. (4) Whilst autoimmune myopathy can start months after initiation, it typically occurs after years of being on a statin. (5)

The average maximum CK level may help in making a distinction. The CK level in immune mediated myopathy ranges between 958 and $450000 \mathrm{IU} / \mathrm{L}$. (5) Cases of rhabdomyolysis is extremely rare in self-limited statin myopathy. (6) Anti HMGCR antibodies are the most specific test available for the diagnosis of statin associated autoimmune myopathy. (7) The case, however to make the diagnosis purely on this antibody level has not been made. MRI changes in both conditions include oedema, muscle atrophy and fatty infiltration and therefore are unhelpful in making a distinction. (8)Muscle biopsy also has its limitation in making the distinction. In one case series 100\% of HMGCR antibodies had myofiber necrosis with around $20 \%$ having endomysial and perivascular imflammation. (8) Patients with rhabdomyolysis in the setting of statin myopathy also have necrotic and degenerating myofibers. In less severe cases one can see cytochrome oxidase-negative fibers, increased lipid stores and vacuolization of the T-tubules. (9) In our patient the absence of myofiber necrosis would make the case for autoimmune myopathy less strong.

Immunosuppresive therapy with prednisolone and a second agent (Methotrexate or intravenous immunoglobulin (IVIG)) have been shown to improve outcome. (5) An important point to raise is that it has been shown around 50$60 \%$ of patients will have a relapse in symptoms when treatment is ceased. (5) This may be the case in our patient's symptoms worsening weeks after her IVIG. It is important to recognised acute fulminant immune mediated myopathy associated with statin therapy. Strong clinical suspicion, cessasion of offending statin, muscle biopsy and prompt treatment with immunomodulatory treatment and antibody testing is strongly advocated. Tests can often take up to weeks to help with the diagnosis and therefore early treatment with immunosuppression would have to be based on the evidence of the clinical situation at presentation.

\section{REFERENCES}

[1] Baigent C, Blackwell L, Emberson J, et al. Efficacy and safety of more intensive lowering of LDL cholesterol: a metaanalysis of data from 170,000 participants in 26 randomised trials. Lancet 2010;376:1670-1681. 
[2] Bruckert E, Hayern G, Dejager S, et al. Mild to moderate muscular symptoms with high- dosage statin therapyin hyperlipidemic patients-the PRIMO study. Cardiovasc Drugs Ther 2005;19:403-414.

[3] Needham M, Fabian V, Knezevic W, et al. Progressive myopathy with up-regulation of MHC-1 associated with statin therapy. NeuromusculDisord 2007;17:194-200.

[4] Hansen KE, Hildebrand JP, Ferguson EE, et al. Outcomes in 45 patients with statin associated myopathy. Arch Intern Med 2005;165:2671-2676.

[5] Grable-Esposito P, Katzberg HD, Greenberg SA, et al. Immune-mediated necrotizing myopathy associated with statins. Muscle Nerve 2010;41:185-190.

[6] Graham DJ, Staffa JA, Shatin D, et al. Incidence of hospitalized rhabdomyolysis in patients treated with lipid lowering drugs. JAMA 2004;292:2585-2590.

[7] Mohassel P, Mammen A. Statin-Associated autoimmune myopathy and anti-HMGCR Autoantibodies.

[8] Mammen AL, Chung T, Christopher-Stine, et al. Autoantibodies against 3-hydroxy-3-methylglutaryl-coenzyme a reductase (HMGCR) in patients with statin-associated autoimmune myopathy. Arthritis Rheum 2011;63:713-721.

[9] Mohaupt MG, Karas RH, Babiychuk, et al. Association between statin-associated myopathy and skeletal muscle damage. Can Med Assoc J 2009;181:E11-18. 